\title{
To Study the Stress among Adolescent
}

\author{
Dr. Santosh $\operatorname{Kumar}^{1 *}$
}

\section{ABSTRACT}

The current study reveals To Study the Stress adolescent there is the significant deference in the level of stress between primary and high school students, high school students are have more stress adolescents there is difference between male and female students there is no significant deference in the level of male and female students.

\section{Keywords: Stress, Adolescent, High School}

Weiten et al (2009) propose that stress that stress is an epidemic in the 21st century that commonly affects of us on a daily basis, when considering minor s from environmental conditions such as noise and pollution; Even though saving gadgets and self-help tools have increased substantially in the 20th century and people have more control over their lives, there is an increase of society and people have less free time. "We well define stress as any instances that threaten or are perceived to threaten one's well-being and hereby tax one'-s coping abilities "for example a person can no longer meet journal demands such as work or relationships they feel they do not have the eye beholder.

Stress is a part of day-to-day living. It is a common human phenomenon and of life as a college student. As college students you may experience stress beating academic demands, adjusting to a new living environment, or developing hand ships.

The stress you experience is not necessarily harmful. Mild forms of stress can act. as a motivator and energizer. However, if your stress level is too medical and social problems can result.

Stress affects most people in some way. Acute (sudden, short-term) stress ads to rapid changes throughout the body. Almost all body systems (the heart and good vessels, immune system, lungs, digestive system, sensory organs, and brain). ear up to meet perceived danger.

\footnotetext{
${ }^{1}$ Asst. Professor, Maharanis Arts, Commerce and Management College For Woman. Bangalore, India *Responding Author

(C) 2016 I S Kumar; licensee IJIP. This is an Open Access Research distributed under the terms of the Creative Commons Attribution License (http://creativecommons.org/licenses/by/2.0), which permits unrestricted use, distribution, and reproduction in any Medium, provided the original work is properly cited.
} 


\section{To Study the Stress among Adolescent}

Stress is simply a reaction to a stimulus that disturbs our physical or mental bruin. In other words, it's an omnipresent part ,of life. A stressful event cans either "fight-or-flight" response, causing hormones such as adrenaline and cal to surge through_ the body. A little bit of stress, known as "acute stress,"

This handout describes different ways of coping effectively with stress. In $r$ to cope effectively, it is first important to know what situations you find and what the experience of stress is like for you. Specific strategies with stress are suggested, such as using relaxation techniques, talking with iris, exercising, and creative expression.

\section{What is Stress?}

Stress is a general term applied to venous mental and physiological "' stress experienced by people feel in their lives.

Stress a term people often use to describe a "feeling of pressure, strain, or ion. People often say that they are "under stress" or feel "stressed out" when a dealing with challenging situations or event. Everyone encounters stressful actions. Sometimes the stress comes from something positive (like a new job, apartment, or new relationship) and sometimes from something negative.

The condition of stress has two components: physical, involving direct or bodily challenge and psychological, involving how individuals receive circumstances in their lives. These components can be examined in three one approach focuses on the environment, describing stress as a stimulus. see this in people's reference to the source or cause of their tension as being an $t$ or set of circumstances such as having "a high-stress job." Physically or psychologically challenging events or circumstances are called stressors.

Researchers who follow this approach study the impact of a wide range of stressors, including (1) catastrophic events, such as tornadoes and earthquakes. (2) ' or life events, such as the loss of a loved one or a job, and (3) chronic substances, such as living with severe pain from arthritis.

\section{CONCEPTUAL FRAME WORK}

\section{Concept of Stress:}

It should be obvious from the opening Case why stress has been called an ease of civilization.

The rapid pace of our lives, overcrowded living conditions too many ands on our time interferences with our personal ambitions and frustrating job conditions all contribute to the modern stress equation; But would we be better off out stress? That would be a life without challenge -no difficulties to surmount new fields to conquer, no reason to sharpen our wits are improving our abilities. Stress is an unavoidable part of living, because every organism faces

(C) The International Journal of Indian Psychology, ISSN 2348-5396 (e) | ISSN: 2349-3429 (p) | 100 


\section{To Study the Stress among Adolescent}

challenges its environment and from its own needs. These challenges are "problems" it t solve if it is to survive and thrive.

Stress is the pattern specific and non-specific responses and orgasm - access to stimulus events that disturb equilibrium and tax or exceed its ability to.

The stimulus events. Include a large variety of external and internal additions that collectively are called stressor. A stressor is a stimulus event that stresses a demand on an organism for some kind of adaptive response. The stress

Ones are composed of a diverse combination of reactions on several levels. Including physibl6gical, behavioral, emotion all and cognitive changes.

Doubt you have observed that some people experience one stressful event after there and do not break down. while others are seriously upset by every low-level stress. This happens because the effect of most stressors is not a direct one, but is

The cognitive appraisal of a stressor is one such moderator variable is it viewed as a threat or a edge? The resource that is available to deal with that stressor is another Streator, variable.

\section{Role of Cognitive Appraisal}

Before a stress response begins, a demand on the organism (stressor) must cognized on some level and evaluated. Cognitive appraisal plays a central role. Defining the situation what the demand is, how big a threat it is, what resources has for meeting it, and what strategies are appropriate. Some stressor, such as injury or finding one's house on fire are seen as threats by almost anyone, many .other stressors can be defined in various ways, depending on our overall situation, the , the relation of this particular demand to our central goals, our : pretence for dealing with it , and our assessment of our competence. The action that causes acute distress for me may be all in a day's work for you.

Our appraisal of a stressor and of our resources for meeting it can be as Portent as the actual stressor in determining our conscious experience, what ting strategies we still see as appropriate, and how successful we still be. If we 11e a stressor as too much for us to deal with, we create a self-fulfilling Percy we are likely to fail even if objectively we are capable of dealing quietly with the demand. Doctors have long known that a parent's attitudes can s important as the physical condition in determining the course of the illness.

Cognitive appraisal may define a stressor as an interesting new challenge will be fun to test oneself against instead of as a threat. The experience may be of exhilaration, of being psyched up anticipating achievement.

(C) The International Journal of Indian Psychology, ISSN 2348-5396 (e)| ISSN: 2349-3429 (p) | 101 


\section{To Study the Stress among Adolescent}

\section{Psychological Stress Reactions}

Our physiological stress reactions are autocratic \& predictable, built-in souses over which we normally have no conscious control. Not so our psychological reactions. They are learned and are heavily dependent on our captions and interpretations of the world and of our capacity for dealing with it. Include behavioral, emotional, and cognitive aspects.

\section{Avowal Patterns}

The behavior of a person under stress depends in part on the level of stress befriended. Mild stress activates and intensifies biologically significant. Visors, Mild stress makes an organism more alert; energies are focused \& performance may improve. Positive behavioral informed, becoming vigilant to access of threat, seeking protection \& $\cdot$ support from others, \& learning better studies \& coping skill.

\section{Life is Little Hassles}

Life is filled with low -level frustration. Your pencil breaks during an exam, get stuck in traffic, or you forget to set your alarm clock for an important appointment. To what extent do these minor irritations pile up to become stressors at play havoc with your health? The answer is to a bigger extent than you might again.

A psychiatrist distributed 100 questionnaires to the faithful waiting for the 2 A.M "bullet" train from Long island into Manhattan. From the 49 completed stationeries returned, it was determined that these average commuters had just doped down their breakfast. in less than 11 minutes, if. at all; were preparation 3 hours each day in transit; and in 10 years had logged about 7500 hours of time assuming two week vacations and no time off for illness. Two thirds of commuters believed their family relations were impaired by their commuting. nine percent experienced fatigue, 47 percent were filled with conscious 28 percent were anxious, and others reported headaches, muscle pains, rejection, and other sympturns of the long-term consequences of beating the rat e in the city by living in the country. (F. Charaton, personal communication, ring, 1973).

\section{Catastrophic Events}

Dining and dancing in a beautiful setting on a Friday evening sounds like a prescription for relieving the stress of a hard week of work. Unfortunately it became, instead, prescription for a disaster, creating great stress, when, in 1982 two aerial walk ways collapsed into the lobby of a hotel in Kansas City, Missouri. Immediately affected were the 2000 people who were attending a tea dance, more than 300 of whom were killed or injured. Also experiencing stress were 1000 rescue workers, who worked more than 10 hours just to get through the rubble to all the victims. Another 5000 people were less directly affected: workers at the hotel, personnel at hospitals in the area, and friends and families of victims (Gist \& Stolz, 1982). No count could be made of those who were affected in the immediate community and to television viewers across the nation, 


\section{To Study the Stress among Adolescent}

as people tried to deal with the senselessness of the event and the anxieties it created about the possibility of other such disasters elsewhere.

Researchers have found that five stages occur predictably in people's responses to disasters:

Typically, there is a period of shock and even "psychic numbness, "during which people cannot fully compare hand what has happened. The next phase involves what has been called "auturnatic action"; people try to respond to the disaster and may behave adaptively, but with little awareness of their actions and poor later memory of the experience.

In the next stage, people often feel great accomplish-meant and even a positive sense of communal effort for a shared purpose. Also in this phase, people feel weary and are aware that they are using up their reserves of energy. During the next phase, they experience a letdown; their energy is depleted and the impact of the tragedy is finally comprehended and felt emotionally. An extended period of recovery follows, as people try to rebuild and to adapt to the changes brought about by the disaster. (Cohen \& Ahearn, 1980)

Knowledge of these typical reaction stages predicting a model that is helpful in predicting people $\mathrm{s}$ reactions - when disaster strikes, enabling rescue workers to anticipate and help victims deal with the problems that arise. Responses to such varied events as fold to1nadoes, airplane crashes, and factory explosions have all been shown to fit this model of disaster reactions.

\section{Chronic Societal Sources of Stress}

What of environmental stressors that part of the ongoing circumstances of life: overcrowding, economic recession, fear of nuclear war? What cumulative effect do such stressors have on us.

\section{COPING STRATEGIES:}

If living is inevitably stressful and if too much stress can disrupt our lives, and even kill us we need to learn own to cope so that we can survive. Coping refers to attempts to meet environmental demands in order to prevent negative consequences (lazarus and folkman, 1984). There, are many different coping techniques, some of which still is more effective than others for a given person in given situation.

Because animals in the wild inputs adapt biologically to their environment, their mechanisms for coping are coded in their genes and limited by the slow timetable of evolutionary processes. Human beings have a tremendous potential for adapting not only biologically, over generations, but psychologically, within a lifetime even within a short period of time if they want to change.

In this final section of the chapter we will look at a variety of strategies that people use to reduce the amount of stress they experience and to lessen its harmful effects. Some strategies are ones that most of us use naturally and habitually, whereas others are special techniques that can be 


\section{To Study the Stress among Adolescent}

learned. Some strategies are individual ones, to be done on one's own in contrast social strategies depend of the presence of other people.

\section{Problem focused versus emotion focused coping}

Coping strategies can be grouped into two main types, depending on whether the goal is to deal with the problem (proofed- focused) or to lessen the discomfort of it (emotion - focused).

The first main approach includes any strategy to deal directly with the stressor whether through overt action or through realistic problem-solving mental emerged was an indecisive coping style that was likely to promote a sense of personal inadequacy which, in turn, was a source of more depression. (coyne et al.1981)

The ego defense mechanisms discussed in chapter 12 (such as repression, denial of reality, and rationalization) are familiar emotion regulating approaches. Undertake unconsciously to protect us appraise situations in less self-threatening ways. They lead to coping strategies that are essentially aimed at self-protection rather than at solving problems. At times, however, they cause us to distort reality and, when overused, can lead to maladaptive coping.

\section{Altering Bodily Reactions}

Stress equals tension for many people. This often means tight muscles, high blood pressure, constricted blood vessels in the brain, and chronic over secretion of hormones. Fortunately; many of these tension responses can be controlled by variety techniques some ages old, some quite new.

\section{Relaxation}

Relaxation through meditation has ancient roots in many parts of the world. For centuries in eastern cultures, ways to calm the mind still the body's tensions have been practiced. Today Zen discipline and yoga exercises from Japan and India are part of daily life for many people both there and, increasingly, in the west. In our own culture, a growing number of people have been attracted to work shops and therapy in relaxation training and to various forms of meditation.

Just as stress is the nonspecific response of the body to any demand made on it, there is growing evidence that complete relaxation is a potent anti's tress, response. The relaxation response is a condition in which muscle tension, cortical.

\section{Modifying Cognitive Strategies:}

A powerful way to handle stress more adaptively is to change our evaluations of stressors and our self-defeating cognitions about the way we are dealing with them. We need to find a different way to think about a given situation, our role in it and the causal attributions we make to explain the undesirable outcome. 


\section{To Study the Stress among Adolescent}

\section{Reappraising Stressors:}

The close connection between cognitive appraisal and the primary of autonomic nervous system arousal has been demonstrated in studies where the cognitive appraisal was systematically varied.

When subjects watched an upsetting film showing vivid circumcision rites in a primitive tribe, they were less physiologically aroused when the film had a sound track that either denied the dangers or discussed them in an intellectual, detached way. (Speisman et al., 1964)

In another study, subjects viewing a film of an industrial accident were less aroused if they were "emotionally inoculated" by being warned in advance that it was coming and given a ·chance to imagine the threatening scenes beforehand. The is cognitive preparation, which gave them an opportunity to rehearse mentally both the stress full episode and their coping.

\section{Supportiveness of the environment}

Life in societies is the most powerful weapon 'in the struggle for life thus it was that thousands of years before humans appeared association of animals in social units was preparing the way for human society (chapin, 1913)

We all cope with stress as individuals, but for a lifetime of effective coping and for the continued success of our species, it is necessary for us to band together with our families, friends, and neighbors (at home and throughout our small planet). Isolation can lead to inadequate coping and is itself the cause of much stress. Much contemporary research points to the improvement in coping that can come from being part of a social support network and from living and working in a healthy environment.

\section{Social support network}

Social support refers to the resources provided by other persons this resources can include material- aid socio-emotional support and informational aid the persons who can provide these resources for an individual are those with whom he are she has significant social relationships such as family members friends coworkers and neighbors

There is now a sizable body of evidence showing that the presence of social support makes people less vulnerable to stress. When people have other people they can turn to, they are better able to handle job stressors, unemployment, marital.

\section{REVIEW OF LITERATURE}

1. Hamiton, Sandra, Fagot Beverly (1998)

Chronic stress and coping styles: A comparison of male and fem ale undergraduates.

(c) The International Journal of Indian Psychology, ISSN 2348-5396 (e)| ISSN: 2349-3429 (p) | 105 


\section{To Study the Stress among Adolescent}

Male and female coping behaviors were compared in orders it test the theory that men the Use instruments coping strategies more frequently than women, who are thought to use emotion focused coping solutions. We interviewed 51 female and 39 male first year undergraduates by telephone there times a week for 8 weeks for 8 weeks. Using an inventory developed for 28 chronic stressors. Analyses of variance were used to test gender differences in frequency of daily stressors, _ concomitant perceptions of stress, and utilization of problem-solving behaviors. The majority expectations.

\section{Compas, Bullce E. (1987)}

Coping with stress doing childhood and adolescence. In the article. Research on how children and adolescents cope with stress and coping's role in reducing the adverse psychological states associated with stress is reviewed. Child and adolescent coping is reflected in seven different lines of research-infants' responses to maternal separation, social support, interpersonal cognitive problem-solving, coping in achievement contexts, Type A behavior pattern in children, repression-sensitization, and resilience to stress. A variety odd efferent coping resources, styles, and specific strategies are important. As well as attempts to deal with adverse emotions associated with stress.

3. Tein. Jemi-yun; sandier, Irwin $\mathbf{N}$;zautra alex j. (2000)

Stressful life events, psychological distress, coping, and parenting of divorced mothers: A longitudinal study.

From the journal of family psychology this was a prospective longitudinal study of the relationship among life stress, psychological distress, coping, and parenting behaviors in a sample $\cdot$ of divorced custodial mothers. First, the differential effects of major events and daily stressors on psychological distress and parenting were explored. Second, the meditational links among stress, distress, and 3dimensions of parenting behaviors were studied. Third, 3coping strategies were studied as moderators of the relationship between distress and parenting. The results showed that results showed that both major and small events had significant effects on parental distress, with effects of daily negative events being greater ,than those of .major events. Parental distress mediated the relationship between stressful life events and parental acceptance of their children's behaviors. Parental coping strategies moderated the relationship between mother's psychological distress and mothers discipline practice.

\section{Valentiner, David P.; Foa, Edna B, Riggs, David S,; Gershuny, Beth S(1996)}

Coping strategies and posttraumatic stress disorder in female victims of sexual and nonsexual assault.(From the journal abstract ) The coping behaviors and (posttraumatic stress disorder [PTSD] symptoms of 215 female assault victims (103 rape victims and 112 nonsexual assault victims) were assessed within 2 weeks following the assault (time), and 133 of them (62\%)were followed up 3 mo later (time 2) . post trauma symptom severity significantly decreased during the 3-mo study period, but PSTD severity levels at times 1 to 2 were highly correlated. Three 


\section{To Study the Stress among Adolescent}

constructed on the basis of exploratory factor analyses: mobilizing support, positive distancing, and wishful thinking. Three mo post assault, rape victims showed higher levels of wishful thinking and PSTD their nonsexual assault victims. Wishful thinking three mo post assault, rape victims showed higher levels of wishful thinking association and positive distancing a negative association with PTSD severity, control1ing for assault rape, initial levels of PTSD severity, and other coping strategies.

\section{METHODOLOGY}

\section{Statement Of The Problem:}

- To study the stress among adolescents.

\section{Variables:}

\section{Independent variable}

a) Adolescents

b) Gender Male and Fem ale

II. Dependent variable

Stress

\section{Objectives:}

1. To study the level of stress among adolescents.

2. To study the level of coping strategies among adolescents.

3. To study the level of coping strategies. among male and female adolescents.

4. To student the level of- stress among male and female adolescents.

\section{Hypothesis:}

1. There is significant difference in the level of stress between adolescents.

2. There is significant difference in the level of coping strategies between adolescents.

3. There is significant difference in the level of coping strategies between male and female students.

4. There is significant difference in the level of stress male and female students.

\section{Tools:}

\section{Singh personal stress source inventory:}

Singh personal stress source inventory is - developed by Arunkumar Singh and -Ashish Kumar Singh and Arpana sing. Which is consisting of 35 items distributed dimension of Singh personal stress source inventory.

The inventory has three response categories namely seldom, sometime, frequently.

The scoring of SPSSI is - very simple. Every item marked as seldom by the tested is given a score of (1), marked as sometime item is given a coat of (2) and marked as frequently a score of (3) 


\section{To Study the Stress among Adolescent}

unmarked items are given a score of zero. Higher the score, the higher is the magnitude of personal stress likewise, lower the score, lower is the magnitude of personal stress, the maximum score on SPSS is 105.

\section{Coping strategies scale:}

Coping strategies scale is developed by prof. A.K. Srivastava which is consisting of 50 items distributed dimension of coping strategies scale. The inventory has five responses categories namely Never, Rarely, Sometimes, Most of the time, Almost always.

The scoring of coping strategies scale. Every item marked as Never item is given a score of (0), marked as Rarely item is given a score of (1 ), marked as Sometimes item is given a score (2). Marked as most of the time item is given a score (3) and marked as Almost always item is given a score (4).

High scores on Avoidance coping strategies would indicate deficient or dysfunctional coping, and low score would indicate efficient or functional coping.

\section{DISCUSSION AND CONCLUSION}

\section{Discussion:}

The present's Studding, designed, to study the stress and coping strategies among adolescents. There are four hypothesis were formulated to test the present study result are discussed in the following table.

Table No. 01, Showing The Level Of Stress Among Adolescents.

\begin{tabular}{|c|c|c|c|}
\hline Faculty & Men & SD & t-value \\
\hline Primary & 60.04 & 5.28 & \multirow{2}{*}{$5.36^{* *}$} \\
\hline High school & 65.05 & 4.82 & \\
\hline
\end{tabular}

**significant at 0.01 level

Table no: 01 showing the level of stress among primary and high school The mean score of primary students is 60.4 and SD us 5.28 and that of high school student is 65.5 and 4.82. The calculated t-value is 5.36 which is significant at 0.01 level of significance. This indicates that there is significant difference in the level of stress between primary and high school students. Primary student are having more stress compare to high school students.

Table No. 02, Showing The Level Of Coping Strategies Among Primary And High School Students

\begin{tabular}{|c|c|c|c|}
\hline Faculty & Men & SD & t-value \\
\hline primary & 85.00 & 14.45 & \multirow{2}{*}{$6.43^{* *}$} \\
\hline university & 101.74 & 11.45 & \\
\hline
\end{tabular}

**significant at 0.01 level

(c) The International Journal of Indian Psychology, ISSN 2348-5396 (e)| ISSN: 2349-3429 (p) | 108 
Table no: 01 showing the level of stress among Primary and high school students. The mean score of Primary students is 85 and SD us 14.45 and that of High school student is 101.74 and 11.45. The calculated t-value is 6.43 which is significant at 0.01 level of significance. This indicates that there is significant difference in the level of coping strategies between Primary and .High school students. Primary student are having low coping strategies compare to High school students.

Table No. 03, Showing The Level Of Coping Strategies Among Male And Female Primary And High School Students.

\begin{tabular}{|l|l|l|l|l|l|l|}
\hline & Primary & & & & $\begin{array}{l}\text { High } \\
\text { school }\end{array}$ & \\
\hline & Mean & SD & t-value & Mean & SD & t-value \\
\hline Male & 80.32 & 15.10 & $2.43^{*}$ & 104 & 10.06 & $1.52 \mathrm{NS}$ \\
\hline Female & 89.68 & 11.97 & & 99.48 & 11.00 & \\
\hline
\end{tabular}

*Significant at 0.01 level

Table no: 03 showing the coping strategies among male and female Primary and high school students.

The mean score of Primary male student is 80.82 and SD is 15.10 and that of Primary female student is 89.68 and 11.97 . The calculated t-value is 2.43 which is significant at 0.05 level of significance. This indicates that there is significant difference, in the level of coping strategies between Primary male and Primary female student. Primary female student are having low coping strategies compare to Primary female students.

The mean score of High school male student is 104 and SD is 10.06 and that of High school female students is 99.48 and, 11.00 . The calculated t-value is 1.52 which is not significant at any level. This indicates that there is no significant difference in the level of coping strategies between High school male and High school female students. When mean score is seen the High school female students are having low coping strategies compare to High school male students.

TABLE NO. 04, Showing the level of stress among male and female Primary and high school students.

\begin{tabular}{|l|l|l|l|l|l|l|}
\hline & \multicolumn{3}{|c|}{ Primary } & \multicolumn{3}{c|}{ High school } \\
\hline & Mean & SD & t-value & Mean & SD & t-value \\
\hline Male & 59.72 & 4.41 & $0.90 \mathrm{NS}$ & 66.28 & 5.60 & l.15NS \\
\hline Female & 61.08 & 6.22 & & 64.72 & 3.33 & \\
\hline
\end{tabular}

Table No.04 Showing the level of stress among male and female Primary and high school students

(C) The International Journal of Indian Psychology, ISSN 2348-5396 (e) | ISSN: 2349-3429 (p) | 109 


\section{To Study the Stress among Adolescent}

The mean score of Primary male student is 59.72 and SD is 4.41 and that of Primary female student is 61.0Rand 6.22. The calculated t-value is 0.90 which is not significant at any level. This indicates that there is no significant difference in the level of stress between Primary male and Primary female students when mean score is seen Primary female student are having more stress compare to Primary male students.

The mean score of High school male student is 66.28 and SD is 5.60 and that of High school female student is 64.72 and 3.33. the calculated t-value is 1.15 which is not significant at any level: This indicates that the.re is no significant difference in the

1. level of stress between High school male and High school female students. High school male student are having more stress compare to High school female students.

\section{CONCLUSION:}

1. There is significant difference in the level of stress between Primary and High school students. High school students are having more stress than Primary students

2. There is significant difference in the level of coping strategies between Primary - and High school students. Primary Student are having low coping strategies then High school Students.

3. There is significant difference in the level of coping strategies between Primary male and Primary female students.

4. There is no significant 'difference in the level of coping strategies between High school male and High school female students.

5. There is no significant difference in the level of stress between male and female Primary and high school students.

\section{REFERENCES}

Devid F. Marks, Michael Murray, Brian Evans, Carla Wellig, Cailine, Woodall and Catherinem. Sykes (sage south Asia edition) 2008.

Devid F. Marks, Michael Murray, Brian Evans and Emee Vida Estacio (sage south Asia edition) 2011.

Health psychology, theory, research and practice second edition (2011)

Health psychology, theory, research and practice third edition (2011)

Irwin G.Sarason, Barbara, Sarason: (2005) Abnormal psychology, the problem of maladaptive behavior 11th edition 2005.

Philip G. Zimbardo Stanford university (1988) Psychology and life Twelfth Edition (golden edition) 1988.

Tata McGraw-Hill edition (2006). Los Angeles Health psychology sixth edition (2006)

How to cite this article: S Kumar (2016), To Study the Stress among Adolescent, International Journal of Indian Psychology, Volume 3, Issue 4, No. 56, ISSN 2348-5396 (e) | ISSN: 23493429 (p), DIP: 18.01.010/20160304, ISBN: 978-1-365-23992-2

(C) The International Journal of Indian Psychology, ISSN 2348-5396 (e) | ISSN: 2349-3429 (p) | 110 